\title{
Mesure de la vitesse de sédimentation d'une suspension par absorption de rayons $\mathbf{X}$
}

\author{
M. Aidi ( $\left.{ }^{1}\right)$, F. Feuillebois $\left({ }^{1}\right)$, A. Lasek $\left({ }^{1}\right)$, R. Anthore $\left({ }^{2}\right)$, C. Petipas $\left({ }^{2}\right)$ et X. Auvray $\left({ }^{2}\right)$ \\ (1) Laboratoire d'Aérothermique du C.N.R.S., 4ter route des Gardes, 92190 Meudon, France \\ ${ }^{(2)}$ Laboratoire des Rayons X, Faculté des Sciences et Techniques, B.P. 118, 76134 Mont Saint Aignan, \\ France
}

(Reçu le 12 mai 1989, révisé le 4 septembre 1989, accepté le 5 septembre 1989)

\begin{abstract}
Résumé. - La mesure de l'absorption de rayons $\mathrm{X}$ monochromatiques permet de déterminer à chaque instant la valeur locale de la concentration d'une suspension polydispersée de particules sphériques. A partir de ces données physiques, on peut obtenir la vitesse moyenne de sédimentation dans la partie homogène de la suspension en suivant la variation au cours du temps du volume total de particules situées au-dessus d'un plan donné. Il est alors possible de comparer ce résultat expérimental à la vitesse moyenne de sédimentation théorique valable pour des suspensions homogènes polydispersées (Batchelor et Wen, 1982). Pour des concentrations volumiques entre $5 \%$ et $15 \%$, la vitesse de sédimentation expérimentale est plus grande que la vitesse théorique calculée pour une faible concentration. Il est alors probable que les interactions hydrodynamiques entre trois sphères ou plus puissent être importantes.
\end{abstract}

\begin{abstract}
The local concentration in a polydisperse suspension of spherical particles is obtained as a function of vertical position and time by measuring the absorption of monochromatic X-rays. From these data, the average velocity of sedimentation in the homogeneous part of the suspension is derived by considering the variation with time of total volume of particles located above a given plane. A comparison with the theoretical average velocity of sedimentation valid for homogeneous polydisperse suspensions (Batchelor and Wen, 1982) is then possible. For volume concentrations between $5 \%$ and $15 \%$, the experimental velocity of sedimentation is larger than the theoretical one, valid for a low concentration. The hydrodynamic interactions between three spheres or more might then be important.
\end{abstract}

\section{Introduction.}

1.1 CONDITIONS EXPÉRIMENTALES. - La sédimentation de particules solides dans un fluide visqueux se retrouve dans différents phénomènes naturels et processus industriels : par exemple, en génie civil, le dépôt des sédiments des rivières, la décantation des eaux usées ; en génie chimique, la sédimentation de précipités formés par floculation. Nous nous limitons ici au cas de particules sphériques et solides de diamètre compris entre $10 \mu \mathrm{m}$ et $500 \mu \mathrm{m}$. L'effet du mouvement brownien est en général négligeable pour des particules de cette taille. Nous nous limitons aussi au cas de suspensions peu concentrées, c'est-àdire au cas où la concentration volumique en particules (volume de particules par unité de volume du mélange) est inférieure à $15 \%$.

1.2 EXPÉRIENCES ANTÉRIEURES. - Un certain nombre de résultats expérimentaux sur la sédimenta- tion ont été publiés. Dans la plupart des cas, la suspension était monodispersée. Une revue de ces résultats est donnée par exemple dans la référence [9]. Lorsque les particules sont suffisamment grandes pour que le mouvement brownien soit négligeable, le sommet du nuage de particules qui sédimentent apparaît très nettement. La méthode la plus naturelle pour obtenir la vitesse de sédimentation consiste alors à suivre ce front haut. Par exemple Richardson et Zaki [10] ont utilisé cette méthode pour mesurer la vitesse de sédimentation de particules de rayon compris entre $100 \mu \mathrm{m}$ et $200 \mu \mathrm{m}$ dans un mélange d'huile de silicone et de white spirit dont la viscosité était 0,162 Poiseuille. Ils ont obtenu la corrélation suivante :

$$
\bar{v}_{\mathrm{p}}=v_{\mathrm{ps}}(1-c)^{4,8}
$$

où $v_{\mathrm{ps}}$ est la vitesse de sédimentation d'une particule 
tombant seule et $c$ est la concentration volumique en particules.

1.3 DIFFÉRENCES PAR RAPPORT AUX EXPÉRIENCES ANTÉRIEURES. - Dans les expériences présentées ici, les suspensions sont polydispersées. Dans ce cas, la différence de vitesse de sédimentation entre les différentes particules provoque un étalement du front haut et la vitesse de sédimentation ne peut donc plus être déterminée de façon suffisamment précise par la vitesse du front. Il est nécessaire d'observer l'intérieur même de la suspension. Ceci est réalisé au moyen de l'absorption des rayons $\mathrm{X}$.

\section{Technique expérimentale utilisant l'absorption des rayons $X$.}

2.1 Montage ExpÉRIMENTAL. - Le montage expérimental est décrit sur la figure 1. Le faisceau de rayons $\mathrm{X}$ issu de la source $\mathrm{S}$ est diffracté par une lame de quartz de façon à obtenir un rayonnement monochromatique. Des fentes $\mathrm{F}_{1}, \mathrm{~F}_{2}, \mathrm{~F}_{3}$, des filtres Fi et un passage sous vide $P$ situés sur le trajet du faisceau permettent d'éliminer le rayonnement parasite, de régler l'intensité incidente, de diminuer l'atténuation par l'air et d'obtenir un faisceau géométriquement parfait. La cellule $\mathrm{C}$ de mesure est placée le plus près possible du détecteur $\mathrm{D}$ à localisation linéaire de façon à minimiser les erreurs de parallaxes dues à la divergence du faisceau. La cellule $\mathrm{C}$ est haute de $80 \mathrm{~mm}$, large de $8 \mathrm{~mm}$ et épaisse (dans le sens du faisceau) de $6 \mathrm{~mm}$. L'analyse porte sur la partie haute de la cellule dont les 5 premiers millimètres ne peuvent être observés. On prend 200 points de mesure sur la verticale avec une résolution spatiale de $0,2 \mathrm{~mm}$. L'adresse liée à la localisation du photon $\mathrm{X}$ fournie par l'électronique $\mathrm{E}$ du détecteur est stockée dans un analyseur multicanaux A. Compte tenu du temps nécessaire pour transférer un spectre dans le microordinateur $\mathrm{Mi}$, une mesure sur 200 points est enregistrée en $60 \mathrm{~s}$.

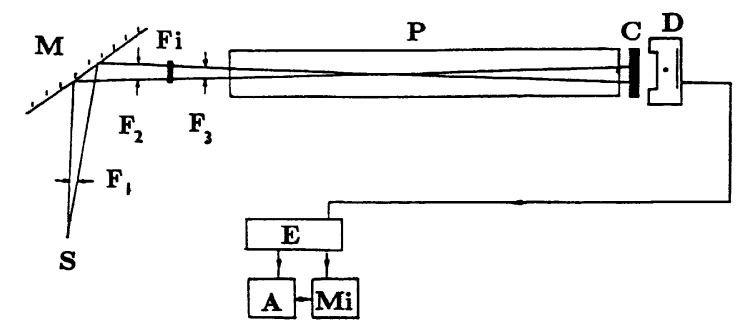

Fig. 1. - Montage expérimental.

[Experimental setup.]

2.2 VAlEuRS PHYSIQUES. - Le fluide est de l'huile de silicone de viscosité dynamique $\eta=1$ Poiseuille et de masse volumique $\rho=970 \mathrm{~kg} / \mathrm{m}^{3}$. Les sphères sont en verre de masse volumique $\rho_{\mathrm{p}}=2700 \mathrm{~kg} / \mathrm{m}^{3}$. On utilise des échantillons dans les gammes de diamètres suivants :

(1) $d=130$ à $240 \mu \mathrm{m}$;

(2) $d=120$ à $180 \mu \mathrm{m}$;

(3) un mélange des deux échantillons précédents.

Les histogrammes des échantillons (1) et (2) représentés sur la figure 2 ont été déterminés par analyse de micrographies optiques projetées sur une table à digitaliser, chaque particule étant analysée individuellement.

\subsection{CONCENTRATION VOLUMIQUE LOCALE EN PAR-} TICULES. - L'atténuation d'un faisceau de rayons $\mathrm{X}$ monochromátique permet de calculer la concentration volumique d'une suspension de particules sphériques homogènes de même nature chimique.

L'intensité $I_{1}$ transmise par la cellule d'épaisseur $\ell$ pleine de liquide pur de coefficient d'atténuation $\alpha \ell$ est :

$$
I_{1}=I_{0} \exp \left(-\alpha_{\ell} \ell\right)
$$

$I_{0}$ étant l'intensité du faisceau incident.

L'intensité $I_{2}$ transmise par une suspension de particules sphériques de diamètre $d$ et de coefficient d'atténuation $\alpha_{\mathrm{p}}$ est :

$$
I_{2}=I_{0} \exp \left(-\alpha_{\ell} \ell\right) \exp \left[-\left(\alpha_{\mathrm{p}}-\alpha_{\ell}\right) \ell_{\mathrm{p}}\right]
$$

$\ell_{\mathrm{p}}$ étant l'épaisseur du solide traversé.

Le coefficient de transmission $T$ est égal à :

$$
T=\frac{I_{2}}{I_{1}}=\exp \left[-\left(\alpha_{\mathrm{p}}-\alpha_{\ell}\right) \ell_{\mathrm{p}}\right]
$$

L'épaisseur $\ell_{\mathrm{p}}$ de particules sphériques traversées est liée à leur diamètre $d$ et à leur nombre $N_{\ell}$ par unité de longueur :

$\ell_{\mathrm{p}}=\left(\frac{2}{3} d\right)\left(N_{\ell} \ell\right)=\left(\frac{2}{3} d\right)\left(N_{\mathrm{v}} \frac{\pi d^{2}}{4} \ell\right)=\ell_{c}$

où $N_{\mathrm{v}}$ et $c$ sont respectivement le nombre de particules par unité de volume et la concentration volumique (volume de particules par unité de volume du mélange). Alors (4) peut s'écrire :

$$
T=\frac{I_{2}}{I_{1}}=\exp \left[-\left(\alpha_{\mathrm{p}}-\alpha_{\ell}\right) c \ell\right] \text {. }
$$

Lorsque la suspension sédimente, l'atténuation est une fonction de la cote $z$ au sein de la cellule et du temps $t: T=T(z, t)$. Alors, $c(z, t)$ peut être calculée à partir de $T(z, t)$ avec (6).

Si les particules sphériques présentent une distribution en tailles, la mesure de l'atténuation donne 
(1)

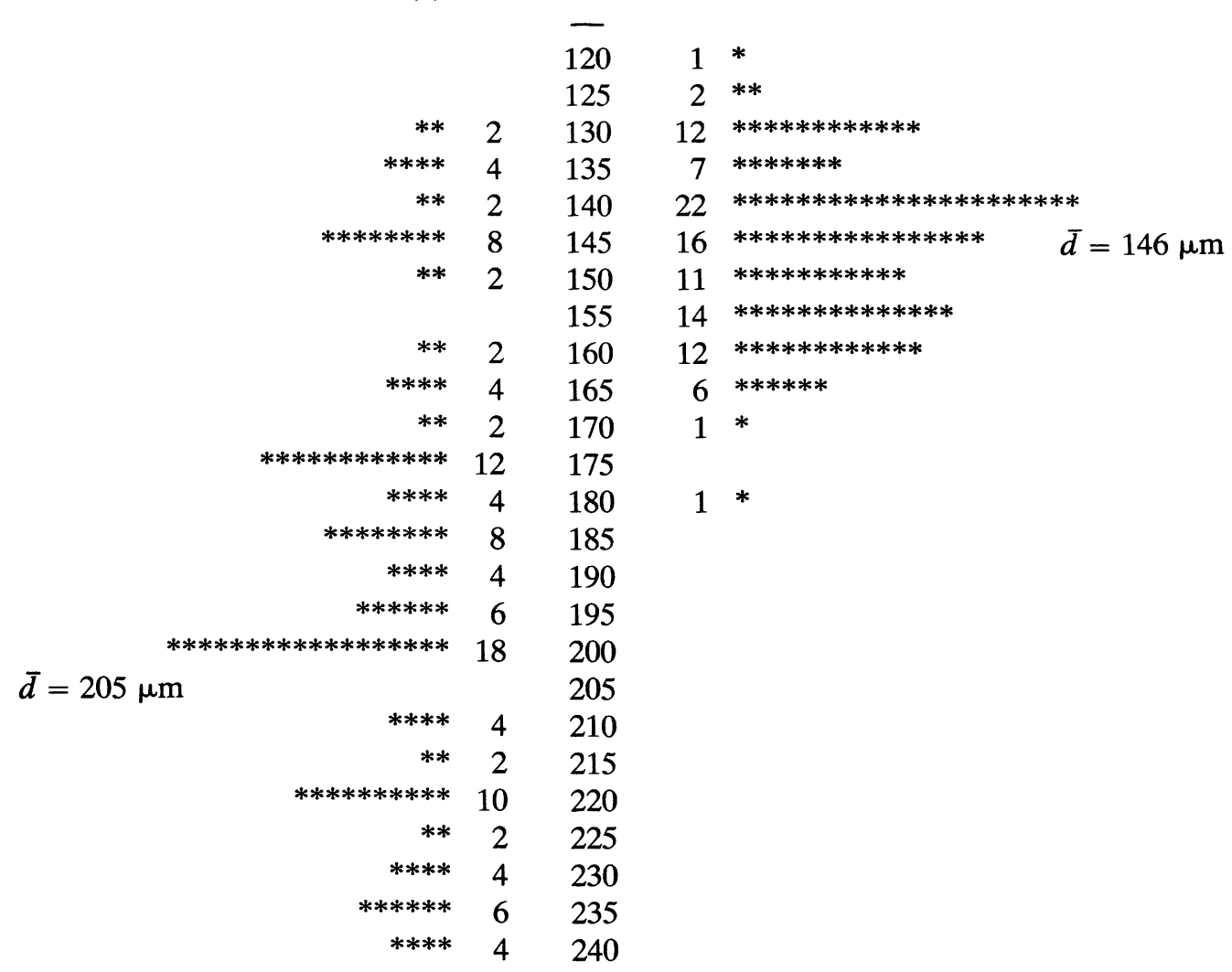

(2) 
$4 \mathrm{~cm}$ de haut), s'ajoute l'incertitude liée à l'échantillonnage en temps (60 s sont nécessaires pour stocker les valeurs aux 200 points de mesure). Ces deux incertitudes sont comparables si la distance parcourue par une particule pendant le temps d'échantillonnage est égale à la distance entre deux points de mesure. Ceci serait vérifié pour des particules de l'ordre de $30 \mu \mathrm{m}$ de rayon qui ont une vitesse de sédimentation de $0,3 \times 10^{-3} \mathrm{~cm} / \mathrm{s}$. Les particules utilisées ici sont plus grosses, de rayons moyens 70 à $100 \mu \mathrm{m}$, et tombent plus rapidement, et c'est l'incertitude liée à l'échantillonnage en temps qui l'emporte. Il est alors inutile d'avoir 200 points de mesure et l'on est amené à utiliser une discrétisation moins fine : en réunissant des voies de mesure, on utilise 51 points de mesure suivant la verticale.

\subsection{COMMENTAIRES SUR LE COMPORTEMENT DU} FRONT HAUT. - Une suspension polydispersée contient des particules plus grosses qui tombent plus vite. D'où un étalement du front. Cependant ceci n'est pas la seule raison possible. La première étape dans la modélisation du comportement du front haut est la théorie de la sédimentation dans une suspension non homogène [8]. Cette théorie s'applique à une suspension monodispersée. On utilise dans cette théorie la probabilité conditionnelle $P\left(\mathbf{x}_{1} \mid \mathrm{x}_{0}\right)$ définie comme la probabilité qu'une sphère soit centrée en un point $\mathbf{x}_{1}$ lorsqu'une autre sphère est déjà centrée en un point $\mathbf{x}_{0}$. Pour obtenir cette probabilité conditionnelle on fait l'hypothèse d'un modèle de sphères dures :

$$
P\left(\mathbf{x}_{1} \mid \mathbf{x}_{0}\right)=\left\{\begin{array}{lll}
N_{\mathrm{v}} & \text { si } & r>d \\
0 & \text { si } & r<d
\end{array}\right.
$$

où $r=\left|\mathbf{x}_{1}-\mathbf{x}_{0}\right|$. Les résultats du calcul montrent qu'un front haut qui est au départ une discontinuité de concentration va ensuite s'étaler et se déformer au cours du temps. Dans un modèle plus complet, le modèle de sphères dures devrait être remplacé par une étude de la variation temporelle et spatiale de la probabilité conditionnelle. Par exemple, les doublets de sphères étant plus rapides que les sphères isolées, le sommet de la suspension sera plus pauvre en doublets après un certain temps. En fait, on ne connaît pas à l'heure actuelle, même pour une suspension monodispersée, le comportement exact du front haut.

Pour ces raisons, nous avons préféré exploiter la partie homogène de la suspension, région pour laquelle une théorie de la sédimentation des suspensions polydispersées est disponible $[3,5,6]$. Comme nous le verrons, les résultats de l'expérience d'absorption des rayons $\mathrm{X}$ peuvent être exploités de façon à calculer la vitesse de sédimentation dans la partie homogène de la suspension.

\section{Partie homogène de la suspension.}

3.1 THÉORIE DES SUSPENSIONS HOMOGÈNES POLYDISPERSÉES. - Considérons deux types de particules sphériques notées $i, j$, respectivement, de diamètres $d_{i}, d_{j}$, et de masses volumiques $\rho_{\mathrm{p} i}, \rho_{\mathrm{p} j}$. La vitesse moyenne de sédimentation d'une particule test de type $i$ est définie comme une moyenne d'ensemble sur la vitesse de la particule test qui interagit avec une autre particule, de type $i$ ou $j$, dont la position dans l'espace est une variable aléatoire. On définit le nombre de Péclet :

$$
\mathrm{Pe}=\frac{1 / 2\left(a_{i}+a_{j}\right)\left|v_{\mathrm{pis}}-v_{\mathrm{pjs}}\right|}{D_{i j}}
$$

où $a_{i}$ ( $a_{j}$ respectivement) est le rayon de la particule $i$ ( $j$ respectivement), $v_{\mathrm{pis}}\left(v_{\mathrm{pjs}}\right.$ respectivement) est la vitesse de la particule de type $i$ ( $j$ respectivement) lorsqu'elle tombe seule dans le fluide,

$$
D_{i j}=\frac{k T}{6 \pi \eta}\left(\frac{1}{a_{i}}+\frac{1}{a_{j}}\right)
$$

est un coefficient de l'ordre de grandeur du coefficient de diffusion d'Einstein ( $\eta$ est la viscosité dynamique du fluide, $k$ la constante de Boltzman et $T$ la température absolue). Lorsque le nombre de Péclet est grand devant l'unité, c'est-à-dire lorsque le mouvement brownien est négligeable, le résultat pour la vitesse moyenne, dirigée suivant la verticale, s'écrit sous la forme suivante [3] :

$$
\bar{v}_{\mathrm{p} i}=v_{\mathrm{p} i \mathrm{~s}}\left(1+S_{i i} c_{i}+S_{i j} c_{j}\right)
$$

où

$c_{i}, c_{j}$ sont les concentrations volumiques des deux types de sphères ;

$S_{i i}=-6,55$ est le coefficient de sédimentation pour une suspension monodispersée ;

$S_{i j}$ est une fonction du rapport des masses volumiques relatives

$$
\gamma=\frac{\rho_{\mathrm{p} j}-\rho}{\rho_{\mathrm{p} i}-\rho}
$$

et du rapport des diamètres

$$
\lambda=\frac{a_{j}}{a_{i}} .
$$

Dans (12), $\rho_{\mathrm{p} i}, \rho_{\mathrm{p} j}$ sont les masses volumiques des deux types de particules et $\rho$ est la masse volumique du fluide.

La formule (11) peut s'étendre à $N$ types de sphères en sommant le terme en $j$ sur tous les types de sphères différents de $i$ :

$$
\bar{v}_{\mathrm{p} i}=v_{\mathrm{p} i s}\left(1+S_{i i} c_{i}+\sum_{\substack{j=1 \\ j \neq i}}^{N} S_{i j} c_{j}\right) .
$$


Les résultats ci-dessus s'appliquent à des types $i, j$ de sphères tels que les diamètres et les masses volumiques soient bien différents. Pour des sphères presqu'identiques, c'est-à-dire lorsque $\lambda \rightarrow 1$ et $\gamma \rightarrow 1$, il existe une singularité de $S_{i j}$ qui n'a pas été étudiée dans tous les cas possibles. En effet, pour $|\gamma-1| \ll|\lambda-1| \ll 1$, le résultat est $S_{i j} \simeq-5,6$, qui est différent de la valeur $S_{i i}=-6,55$ obtenue pour les suspensions monodispersées; pour $|\gamma-1| \sim|\lambda-1| \ll 1$, le coefficient de sédimentation n'a pas été calculé. Cette singularité peut s'expliquer de la façon suivante. Le déplacement relatif de deux sphères presqu'identiques est très petit car deux sphères identiques qui interagissent tombent à la même vitesse. On voit alors que dans l'expression (9) du nombre de Péclet le dénominateur peut devenir du même ordre de grandeur que le numérateur. Le mouvement brownien, quoique faible, devient important dans le mouvement relatif des sphères et donc dans la détermination de la probabilité conditionnelle de présence d'une sphère par rapport à une autre. Les résultats calculés pour un grand nombre de Péclet (9) ne sont alors plus valables.

3.2 Discussion. - Expérimentalement, il est plus facile d'obtenir des sphères de masses volumiques presqu'égales que de diamètres presqu'égaux. Le rapport des masses volumiques relatives, $\gamma$, et le rapport des diamètres, $\lambda$, satisfont généralement à $|\gamma-1| \ll|\lambda-1| \ll 1$. Le .nombre de particules dont les rayons sont suffisamment proches pour que $|\gamma-1| \sim|\lambda-1| \ll 1$ est alors petit devant le nombre total de particules de la suspension ; ces particules ont donc une contribution négligeable à la vitesse moyenne de sédimentation [1].

\subsection{MÉTHOde EXPÉRIMENTALE. - L'absorption} des rayons $\mathrm{X}$ donne la concentration locale en particules, en fonction de la position verticale et du temps. Le volume de particules $V_{\mathrm{p}}(t)$ situé entre un plan de contrôle donné à la cote $z_{\mathrm{p}}$ et le sommet de la partie irradiée de la cellule à la cote $H_{\mathrm{v}}$ peut se déduire des résultats expérimentaux par la formule suivante :

$$
V_{\mathrm{p}}(t)=\mathcal{A} \int_{z_{\mathrm{p}}}^{H_{\mathrm{v}}} c(z, t) \mathrm{d} z
$$

La quantité obtenue est une fonction du temps $t$. $\mathcal{A}$ est l'aire d'une section horizontale de la cellule. Lorsque le sommet du nuage de particules a traversé le plan situé en $H_{\mathrm{v}}$, l'équation (15) représente également le volume total de particules qui se trouvent au-dessus du plan de contrôle situé en $z_{\mathrm{p}}$.

Il est utile pour la suite de représenter en fonction du temps une quantité qui a la dimension d'une longueur : c'est le volume réduit $z(t)=V_{\mathrm{p}}(t) / \mathcal{A} c_{0}$, où $c_{0}$ est la concentration initiale. $z(t)$ peut aussi s'interpréter comme étant la hauteur au-dessus de $z_{\mathrm{p}}$ qu'aurait la suspension si elle était homogène et de concentration $c_{0}$, c'est-à-dire si le front haut avait une largeur nulle.

A titre d'exemple on a représenté figure 4 le graphe de $z(t)$ pour l'échantillon (1) décrit dans le paragraphe 2.2 , avec une concentration initiale $c_{0}=10 \%$. Le plan de contrôle choisi est situé en $z_{\mathrm{p}}=1,346 \mathrm{~cm}$ et l'on voit qu'une grande partie de la courbe est alors quasiment linéaire. Cette partie correspond au passage de la partie homogène de la suspension au travers du plan de contrôle ; la partie homogène est la région où les concentrations $c_{i}$ en particules de types $i=1 \ldots N$ restent égales aux concentrations initiales $c_{i 0}$, et donc où la concentration totale $c$ reste égale à la concentration totale initiale $c_{0}$. La partie non linéaire de la courbe, pour les temps les plus grands, correspond au passage du front haut dans lequel toutes les concentrations varient. La non-linéarité de la partie haute de la courbe correspond au passage du front de sédimentation dans la zone supérieure de la cellule qui ne peut être observée avec les rayons $\mathrm{X}$.

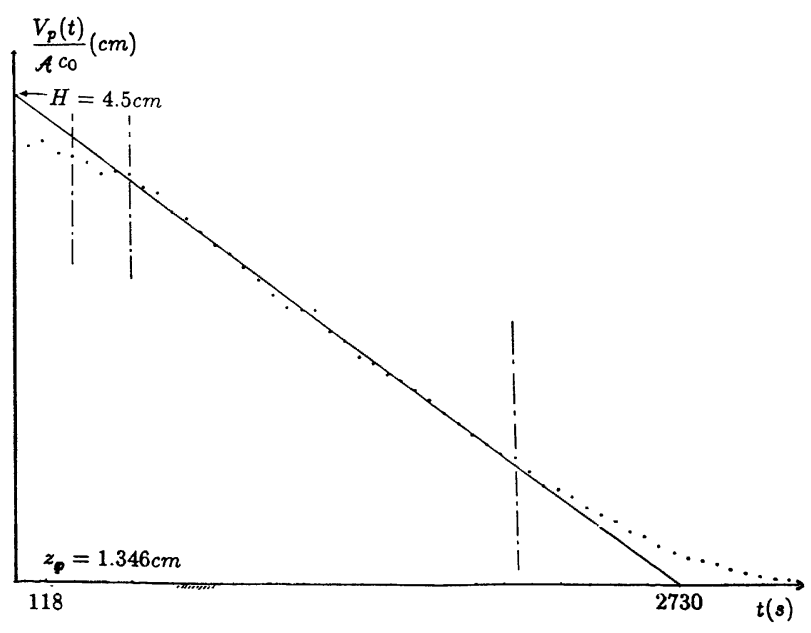

Fig. 4. - Volume réduit de particules situées au-dessus du plan de contrôle $z_{\mathrm{p}}=1,346 \mathrm{~cm}$ en fonction du temps. $H=4,5 \mathrm{~cm}$ est le sommet de la suspension homogène au temps zéro.

[Reduced volume of particles above a given control plane $z_{\mathrm{p}}=1.346 \mathrm{~cm}$ as a function of time. $H=4.5 \mathrm{~cm}$ is the top of the homogeneous suspension at time zero.]

Par définition, la vitesse moyenne de sédimentation dans la partie homogène de la suspension est la dérivée par rapport au temps du volume réduit de particules situées au-dessus du plan de contrôle, dans la région où ce volume réduit varie linéairement en fonction du temps. Cette quantité est obtenue pratiquement en mesurant la pente de la partie linéaire de la courbe tracée figure 4 . La vitesse 
moyenne ainsi définie est le flux total de particules à travers le plan de contrôle. On peut écrire aussi que c'est la somme des flux des différents types de particules :

$$
\bar{v}_{\mathrm{p}}=\frac{\mathrm{d}\left(z-z_{\mathrm{p}}\right)}{\mathrm{d} t}=\frac{1}{c} \sum_{i=1}^{N} \bar{v}_{\mathrm{p} i} c_{i} .
$$

Si l'on introduit l'expression théorique (14) de la vitesse moyenne de chaque type de particules, la vitesse moyenne de sédimentation (16) peut s'écrire sous la forme suivante [1] :

$$
\bar{v}_{\mathrm{p}}=v_{\mathrm{ps}}(1+S c)
$$

où :

$$
v_{\mathrm{ps}}=k \sum_{i=1}^{N} d_{i}^{2} \chi_{i} ; \quad k=v_{\mathrm{p} i \mathrm{~s}} / d_{i}^{2}
$$

et

$$
S=\frac{\sum_{i=1}^{N} d_{i}^{2} \chi_{i} \sum_{j=1}^{N} S_{i j} \chi_{j}}{\sum_{i=1}^{N} d_{i}^{2} \chi_{i}}
$$

sont des fonctions des taux de concentration en particules de type $i$ dans le mélange :

$$
\chi_{i}=c_{i} / c \text {. }
$$

La constante suivante apparaît aussi dans l'équation (18) :

$$
k=\frac{v_{\mathrm{p} i \mathrm{~s}}}{d_{i}^{2}}=\frac{1}{18}\left(\frac{\rho_{\mathrm{p} i}-\rho}{\eta}\right) g .
$$

Le coefficient de sédimentation $S$ est une moyenne pondérée des coefficients de sédimentation théoriques $S_{i j}$. Dans nos expériences, les masses volumiques des particules sont pratiquement identiques. Nous utilisons alors les valeurs théoriques $[5,6]$ de $S_{i j}$ données dans le cas $\mathrm{Pe} \gg 1, \gamma=1$ et pour différentes valeurs de $\lambda$. Il est intéressant de noter que, bien que la gamme de variation de $S_{i j}$ soit assez grande, $S$ varie peu avec $\lambda$ :

- si $\lambda=1, S_{i j}=-5,6$, alors $S=-5,6$;

- si $\lambda=1 / 8, \quad S_{i j}(\lambda)=-3,68$ et $S_{j i}(\lambda)=$ $S_{i j}(1 / \lambda)=-78,53$, alors $S=-4,83$.

Nous avons choisi $\lambda=1 / 8 \mathrm{car}$, parmi les valeurs utilisées dans [5], c'est la plus proche des bornes de nos histogrammes.

En conclusion, la vitesse moyenne de sédimentation définie en (16) et mesurée par la méthode expérimentale présentée peut être comparée à une vitesse moyenne obtenue à partir des résultats théoriques.
3.4 VALEURS EXPÉRIMENTALES DU COEFFICIENT DE SÉDIMENTATION. - Nous avons déterminé la vitesse moyenne de sédimentation $\bar{v}_{\mathrm{p}}$ à partir de la pente de la courbe représentant $z(t)$ (voir par exemple la figure 4) pour la gamme de concentration : $5 \% \leqslant c_{0} \leqslant 15 \%$. Les suspensions utilisées étaient des échantillons de types (1), (2) et (3) décrits au paragraphe 2.2. Dans le cas de l'échantillon (3), mélange des échantillons (1) et (2), nous avons utilisé différentes valeurs du rapport

$$
\alpha=\frac{c_{(1)}}{c_{0}}
$$

où $c_{(1)}$ est la concentration en particules ayant l'histogramme (1) dans le mélange et $c_{0}$ est la concentration totale du mélange.

La valeur expérimentale de $v_{\mathrm{ps}}$, que nous notons $\left(v_{\mathrm{ps}}\right)_{\mathrm{c}}$, est obtenue en extrapolant la courbe $\bar{v}_{\mathrm{p}}$ fonction de $c$ jusqu'à $c=0$. Cette valeur est comparée au résultat théorique, que nous notons $\left(v_{\mathrm{ps}}\right)_{\mathrm{t}}$, calculé avec les équations (17)-(21). Les résultats sont présentés dans le tableau I.

Tableau I. - Valeurs expérimentales et théoriques de $v_{\mathrm{ps}}$ et valeurs expérimentales de $S$ en fonction de $\alpha$.

[Experimental and theoretical values of $v_{\mathrm{ps}}$ and experimental values of $S$ versus $\alpha$.]

\begin{tabular}{|c|r|r|r|r|c|}
\hline$\alpha$ & \multicolumn{1}{|c|}{0} & 0,33 & 0,50 & 0,67 & 1 \\
\hline$\left(v_{\mathrm{ps}}\right)_{\mathrm{c}} \times 10^{5} \mathrm{~m} \mathrm{~s}^{-1}$ & 1,81 & 2,43 & 2,73 & 3,05 & 3,67 \\
\hline$\left(v_{\mathrm{ps}}\right)_{\mathrm{t}} \times 10^{5} \mathrm{~m} \mathrm{~s}^{-1}$ & 1,95 & 2,52 & 2,81 & 3,09 & 3,66 \\
\hline$S$ & $-3,6$ & $-3,6$ & $-3,5$ & $-3,5$ & $-3,4$ \\
\hline
\end{tabular}

Les variations de $S$ indiquées dans le tableau I sont peu importantes, de sorte qu'il est possible de tracer une seule ligne droite à travers tous les points expérimentaux représentés dans un graphe de $\bar{v}_{\mathrm{p}} / v_{\mathrm{ps}}$ en fonction de $c$, figure 5 . La pente de la droite, représentée en trait continu, est $S=-3,55$. La droite de pente $S=-5,6$, valeur théorique correspondant au cas limite de sphères presqu'identiques, est représentée en pointillé.

\subsection{DisCUSSION DES RÉSULTATS. - On observe sur} la figure 5 que les points expérimentaux obtenus pour $c_{0}=5 \%$ sont plus dispersés qu'aux concentrations supérieures. En effet, le contraste est faible entre les particules en verre et l'huile de silicone contenant de la silice; lorsque les particules sont moins nombreuses, les différences d'intensité des rayons $\mathrm{X}$ en fonction de $c$ sont faibles.

On observe aussi qu'aux concentrations intermé- 


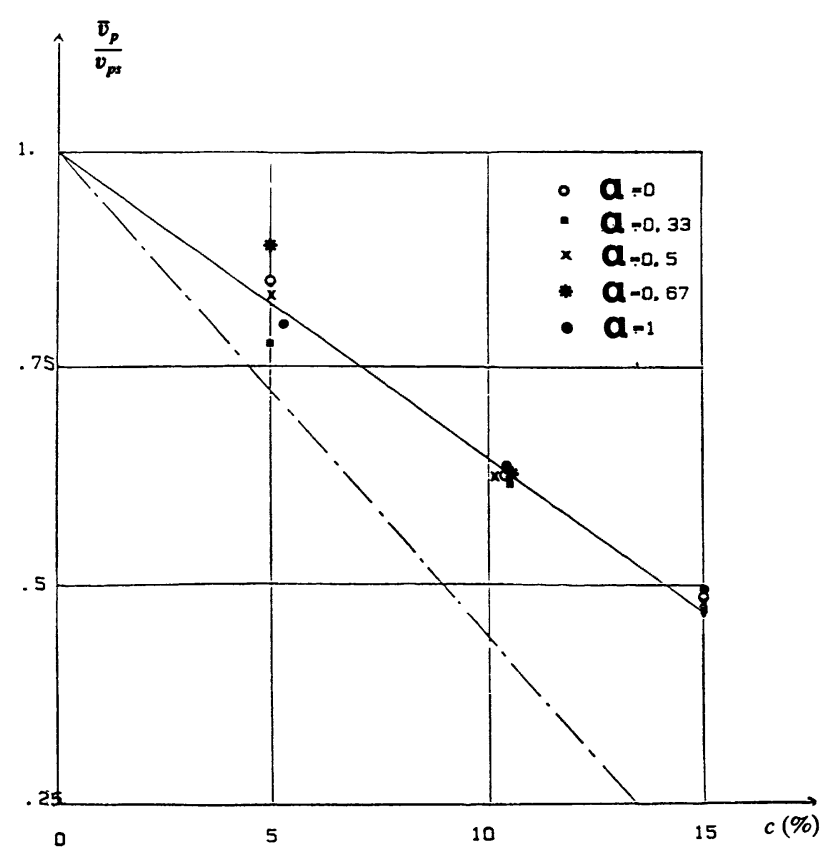

Fig. 5. - Vitesse moyenne de sédimentation réduite en fonction de la concentration volumique totale, pour différentes valeurs du taux $\alpha$ en particules ayant l'histogramme (1).

[Reduced average velocity of sedimentation as a function of the total volume concentration, for various values of the fraction $\alpha$ in particles with histogram (1).]

diaires $(5 \% \leqslant c \leqslant 15 \%)$ la valeur expérimentale du coefficient de sédimentation est supérieure aux bornes théoriques $-5,6 \leqslant S \leqslant-4,83$. Ces bornes ont été obtenues dans le cadre de l'hypothèse de faible concentration volumique et sont basées sur l'étude des interactions hydrodynamiques entre paires de sphères. Le fait que le coefficient expérimental ne se situe pas entre ces bornes théoriques indique donc que les interactions entre trois sphères, ou plus, peuvent devenir importantes.

Des expériences en cours, utilisant un liquide présentant un meilleur contraste avec les particules, permettront d'explorer le domaine des concentrations inférieures à $5 \%$.

D'autres effets physiques pourraient apparaître aux concentrations intermédiaires: en particulier des instabilités ont été observées dans des suspensions bidispersées [4]. Ce ne semble toutefois pas être le cas ici, car de telles instabilités devraient entraîner de fortes dispersions dans les résultats expérimentaux, ce qui n'a pas été observé.

\section{Conclusion.}

Nous avons utilisé la technique d'absorption de rayons $\mathrm{X}$ pour observer une suspension polydispersée de sphères en sédimentation. La vitesse moyenne de sédimentation, définie comme étant le flux de particules à travers un plan de référence, est calculée à partir de la variation au cours du temps du volume de particules situées au-dessus de ce plan. La vitesse moyenne ainsi définie s'exprime en fonction des vitesses moyennes de sédimentation de chaque espèce définies et calculées théoriquement par Batchelor et Wen [5, 6]. En outre, la contribution des particules presqu'identiques, pour laquelle des problèmes théoriques subsistent, se trouve être négligeable. Grâce à cette cohérence entre les différentes vitesses moyennes de sédimentation, une comparaison théorie-expérience est possible.

Avec les particules et le fluide utilisés jusqu'à présent, le contraste aux faibles concentrations $(c<5 \%)$ est insuffisant. Aux concentrations intermédiaires $(5 \% \leqslant c \leqslant 15 \%)$, la valeur du coefficient de sédimentation $S$ trouvé expérimentalement est généralement plus grande que la valeur théorique (qui est valable toutefois pour de faibles concentrations), la valeur moyenne étant $S=-3,5$. Il est alors probable que les interactions hydrodynamiques entre trois sphères, ou plus, puissent devenir importantes. Or, le problème théorique du calcul des interactions hydrodynamiques dans une suspension concentrée polydispersée n'a pas été traité. Il faudrait en particulier déterminer la probabilité d'existence des configurations de triplets (quadruplets, ...) de sphères en fonction des interactions hydrodynamiques.

La méthode expérimentale que nous avons présentée ici pourrait s'appliquer à d'autres expériences utilisant par exemple l'absorption des rayons $\gamma[7]$ ou le temps de parcours des ondes ultrasonores [2].

\section{Remerciements.}

Les auteurs remercient le Professeur P. Haymann pour la détermination des histogrammes et Monsieur Bostel (URA 808, Rouen) pour la réalisation du montage expérimental. 


\section{Bibliographie}

[1] AIDI M., Sur la sédimentation d'une suspension polydispersée homogène ou non, $\mathrm{PhD}$ thesis, Université de Rouen, 1986.

[2] Bacri J. C., Frenois C., Hoyos M., Perzynski R. Rakotomalala N. and Salin D., Acoustic study of suspension sedimentation, Europhys. Lett. 2 (2) 1986, 123.

[3] BAtChELOR G. K., Sedimentation in a dilute polydisperse system of interacting spheres, part. 1: general theory, J. Fluid Mech. 119 (1982) 379.

[4] Batchelor G. K. and Janse Van Rensburg R. W., Structure formation in bidisperse sedimentation, J. of Fluid Mech. 166 (1986) 379.

[5] BAtchelor G. K. and WeN C. S., Sedimentation in a dilute polydisperse system of interacting spheres, part 2 : numerical results, J. Fluid Mech. 124 (1982) 495.

[6] BAtChelor G. K. and Wen C. S., Sedimentation in a dilute polydisperse system of interacting sphe- res, part 2 : numerical results, corrigendum, $J$. Fluid Mech. 137 (1983) 467.

[7] Dunand A. and Soucemarianadin A., Concentration effects on the settling velocities of proppant slurries, Technical Report 14259, Society of Petroleum Engineers, 1985.

[8] Feuillebois F., Sedimentation in a dispersion with vertical inhomogeneities. J. Fluid Mech. 139 (1984) 145.

[9] Feuillebois F., Some theoretical results for the motion of solid spherical particles in a viscous fluid, Multiphase Science and Technology, volume 4, G. F. Hewitt, J. M. Delhaye and N. Zuber Eds (Hemisphere Publishing Co, New York) 1989, pp. 583-798.

[10] Richardson J. F. and ZaKi W. N., Sedimentation and fluidization, part 1, Trans. Instn. Chem. Eng. 32 (1954) 35. 